\title{
Does metformin modify the effect on glycaemic control of aerobic exercise, resistance exercise or both?
}

\author{
Normand G. Boulé • Glen P. Kenny • Joanie Larose • \\ Farah Khandwala $\cdot$ Nicholas Kuzik • Ronald J. Sigal
}

Received: 25 April 2013 / Accepted: 23 July 2013 /Published online: 23 August 2013

(C) Springer-Verlag Berlin Heidelberg 2013

\begin{abstract}
Aims/hypothesis Some previous studies suggested that metformin might attenuate the effects of exercise on glycaemia or fitness. We therefore examined whether metformin use influenced changes in glycaemic control, fitness, body weight or waist circumference resulting from aerobic and/or resistance training in people with type 2 diabetes participating in an exercise intervention trial.

Methods After a 4 week run-in period, participants from the Diabetes Aerobic and Resistance Exercise (DARE) trial were randomly assigned to 22 weeks of aerobic training alone, resistance training alone, combined aerobic and resistance exercise training or a waiting-list control group. Of the 251 randomised, 143 participants reported using metformin throughout the entire study period and 82 reported not using metformin at all.

Results Compared with control, aerobic training led to a significant reduction in $\mathrm{HbA}_{1 \mathrm{c}}$ in the metformin users $(-0.57 \%$, $95 \%$ CI $-1.05,-0.10 ;-6.3 \mathrm{mmol} / \mathrm{mol}, 95 \% \mathrm{CI}-11.5,-1.1)$ but not in the non-metformin users $(-0.17,95 \% \mathrm{CI}-0.78$,
\end{abstract}

N. G. Boulé $(\bowtie) \cdot$ N. Kuzik

Faculty of Physical Education and Recreation, 1-002 Li Ka Shing Centre for Health Research Innovation, University of Alberta,

Edmonton, AB, Canada T6G 2E1

e-mail: nboule@ualberta.ca

G. P. Kenny $\cdot$ J. Larose

School of Human Kinetics, Faculty of Health Sciences,

University of Ottawa, Ottawa, ON, Canada

F. Khandwala

Alberta Health Services, Calgary, AB, Canada

\section{R. J. Sigal}

Departments of Medicine, Cardiac Sciences and Community Health Sciences, Faculties of Medicine and Kinesiology,

University of Calgary, Calgary, AB, Canada
$0.43 ;-1.9 \mathrm{mmol} / \mathrm{mol}, 95 \% \mathrm{CI}-8.5,4.7)$. However, there were no significant differences in the changes in $\mathrm{HbA}_{1 \mathrm{c}}$ (or fasting glucose) between metformin users and non-users in any of the exercise groups compared with control $(p>0.32$ for all metformin by group by time interactions). Similarly, metformin did not affect changes in indicators of aerobic fitness, strength and body weight or waist circumference ( $p \geq 0.15$ for all metformin by group by time interactions).

Conclusions/interpretation Contrary to our hypothesis and to previous short-term studies, metformin did not significantly attenuate the benefits of exercise on glycaemic control or fitness.

Keywords Body mass $\cdot$ Exercise $\cdot$ Fasting glucose $\cdot$ Fitness $\cdot$ $\mathrm{HbA}_{1 \mathrm{c}} \cdot$ Metformin
Abbreviations
DARE Diabetes Aerobic and Resistance Exercise
$\dot{V} \mathrm{O}_{2 \text { peak }} \quad$ Peak oxygen consumption

\section{Introduction}

Exercise and metformin are among the most widely prescribed first-line therapies in type 2 diabetes. Recently, some studies suggested that the glucose-lowering or insulinsensitising effects of exercise may be affected by metformin [1-3]. These studies did not examine whether participants already taking metformin would also have attenuated improvements in glycaemic control following exercise training, as reflected in longer-term indicators of glycaemic control such as $\mathrm{HbA}_{1 \mathrm{c}}$.

Metformin may also alter the effect of exercise training on fitness related outcomes. For example, peak oxygen consumption $\left(\dot{V} \mathrm{O}_{2 \text { peak }}\right)$ was reduced following 7-9 days of treatment with metformin [4], although this did not occur after only a single dose of metformin [5]. 
The primary objective of this study was to examine the association between metformin and improvements in $\mathrm{HbA}_{1 \mathrm{c}}$ following aerobic and/or resistance exercise training in people with type 2 diabetes. It was hypothesised that exercise training would result in smaller reductions in $\mathrm{HbA}_{1 \mathrm{c}}$ in those taking metformin.

\section{Methods}

Participants Previously inactive patients with type 2 diabetes, 39-70 years of age, were recruited. Exclusion criteria included $\mathrm{HbA}_{1 \mathrm{c}}<6.6 \%$ or $>9.9 \%(<49 \mathrm{mmol} / \mathrm{mol}$ or $>85 \mathrm{mmol} / \mathrm{mol})$ and current insulin therapy. Greater details for the methods as well as the primary results of the Diabetes Aerobic and Resistance Exercise (DARE) trial have been published [6].

Design After a 4 week run-in period, participants were randomised to four groups: aerobic training (Aerobic), resistance training (Resistance), combined aerobic and resistance training (Combined) or waiting-list control (Control). Randomisation was stratified by sex and age. The study was approved by the Ottawa Hospital Research Ethics Board and participants gave informed consent (ClinicalTrials.gov registration no. NCT00195884).

Run-in period and exercise interventions Before randomisation, participants entered a 4 week run-in period to assess compliance. Participants were supervised and performed 15-20 min of aerobic exercise and one or two sets of eight resistance exercises. Participants attending $\geq 10$ of the 12 run-in sessions were eligible for randomisation.

Exercise supervision was provided weekly for the first 4 weeks after randomisation, bi-weekly for the next 4 weeks and then every 4 weeks thereafter. Participants exercised three times a week. Aerobic training progressed to $45 \mathrm{~min}$ per session at $75 \%$ of maximum heart rate. Resistance training involved seven exercises on weight machines each session, progressing to two or three sets at the maximum weight that could be lifted seven to nine times. The Combined group did the full aerobic programme plus the full resistance programme. Control participants were asked to revert to prestudy activity levels. They maintained the same dietary intervention and time with the research coordinator/dietician as their exercise group counterparts. They received free 6 month gym memberships after the end of the intervention. In all groups, efforts were made to minimise dietary and medication co-intervention [6].

Assessment of medication use Medication use was assessed at baseline, 3 months and 6 months. Participants were considered to be treated with metformin if they reported taking metformin at all three time points. They were considered not to be treated with metformin if they reported no metformin use at any visit.

Outcome measures The primary outcome was absolute change in $\mathrm{HbA}_{1 \mathrm{c}}$ between baseline and the end of the 6 month supervised exercise period. $\mathrm{HbA}_{1 \mathrm{c}}$ was measured by turbidimetric immunoinhibition. Secondary outcomes included fasting glucose, aerobic fitness, strength, anthropometrics and exercise adherence.

Plasma glucose was measured after a $12 \mathrm{~h}$ fast, at least $48 \mathrm{~h}$ after the last exercise session. $\dot{V} \mathrm{O}_{2 \text { peak }}$ was determined during a maximal treadmill exercise stress test and strength testing involved determining the maximum weight that could be lifted eight times $[6,7]$. Exercise adherence was calculated from electronic membership card use.

Statistical analysis Baseline characteristics of metformin users and non-users were compared with $\chi^{2}$ statistics for categorical variables and Student's $t$ tests for continuous variables. For the primary analysis, we used a linear mixedeffects model for repeated measures over time with $\mathrm{HbA}_{1 \mathrm{c}}$ as the dependent variable. Contrast estimates from the mixed model were calculated for metformin by group by time interaction (Control vs Aerobic, Control vs Resistance, Control vs Combined), with age, sex, BMI and exercise facility as covariates.

\section{Results}

Of the 258 volunteers who were eligible for the study and entered the 4 week run-in phase, $251(97.3 \%)$ met the criteria for random assignment. Of these, there were 3, 12, 7 and 8 dropouts from the Control, Aerobic, Resistance and Combined groups, respectively, during the intervention period. One hundred and forty-three participants reported taking metformin throughout the entire study and 82 reported not taking metformin; see reference [6] for the complete trial flow diagram. The remaining 26 participants were not included in the analyses due to changes in their metformin use during the study period. The mean metformin dose was unchanged from baseline to the end of the interventions $(1,603 \pm 600$ vs $1,654 \pm 616 \mathrm{mg} /$ day). Characteristics of the participants are summarised in Table 1.

As previously reported [6], there was a significant overall reduction in $\mathrm{HbA}_{1 \mathrm{c}}$ in all exercise groups. Compared with Control, in the Aerobic group there was a significant reduction in $\mathrm{HbA}_{1 \mathrm{c}}$ in the metformin users $(-0.57 \%, 95 \% \mathrm{CI}-1.05$, $-0.10 ;-6.3 \mathrm{mmol} / \mathrm{mol}, 95 \% \mathrm{CI}-11.5,-1.1)$ but not in the non-metformin users $(-0.17,95 \% \mathrm{CI}-0.78,0.43$; $-1.9 \mathrm{mmol} / \mathrm{mol}, 95 \% \mathrm{CI}-8.5,4.7)$. Compared with Control, the exercise programme in the Combined group led to significant reductions in $\mathrm{HbA}_{1 \mathrm{c}}$ in both the metformin users $(-1.05 \%, 95 \%$ CI $-1.52,-0.59 ;-11.5 \mathrm{mmol} / \mathrm{mol}, 95 \%$ CI 
Table 1 Baseline characteristics

\begin{tabular}{|c|c|c|c|}
\hline Characteristic & $\begin{array}{l}\text { Non-metformin } \\
\text { users }\end{array}$ & $\begin{array}{l}\text { Metformin } \\
\text { users }\end{array}$ & $p$ value \\
\hline $\operatorname{Sex}(n$, men/women $)$ & $46 / 36$ & $100 / 43$ & 0.036 \\
\hline Age (years) & $53.1(6.9)$ & $54.9(7.1)$ & 0.067 \\
\hline Duration of diabetes (years) & $3.7(3.8)$ & $6.3(4.4)$ & $<0.001$ \\
\hline BMI $\left(\mathrm{kg} / \mathrm{m}^{2}\right)$ & $33.3(6.4)$ & $33.3(5.5)$ & 0.942 \\
\hline Body weight (kg) & $96.1(21.1)$ & $96.8(17.9)$ & 0.804 \\
\hline \multicolumn{4}{|l|}{$\mathrm{HbA}_{1 \mathrm{c}}$} \\
\hline$(\%)$ & $7.47(0.77)$ & $7.78(0.92)$ & 0.011 \\
\hline$(\mathrm{mmol} / \mathrm{mol})$ & $58.1(8.4)$ & $61.5(10.1)$ & 0.011 \\
\hline Fasting glucose (mmol/l) & $8.9(2.3)$ & $9.6(2.3)$ & 0.028 \\
\hline$\dot{V} \mathrm{O}_{2 \text { peak }}\left(\mathrm{ml} \mathrm{kg}^{-1} \mathrm{~min}^{-1}\right)$ & $23.1(4.7)$ & $22.5(4.6)$ & 0.378 \\
\hline
\end{tabular}

Data are presented as mean (SD) except for sex

Baseline characteristics between metformin users and non-users were compared with $\chi^{2}$ statistics for categorical variables and Student's $t$ tests for continuous variables. There were no significant differences between groups (i.e. Control, Aerobic, Resistance and Combined). There were no metformin by group interactions

$-16.7,-6.4)$ and the non-metformin users $(-0.81 \%, 95 \% \mathrm{CI}$ $-1.37,-0.25 ;-8.9 \mathrm{mmol} / \mathrm{mol}, 95 \% \mathrm{CI}-15.0,-2.7)$. Compared with Control, in the Combined group there was a significant reduction in fasting glucose in the metformin users $(-1.47 \mathrm{mmol} / 1,95 \% \mathrm{CI}-2.54,-0.39)$ but not in the nonmetformin users $(-0.52 \mathrm{mmol} / 1,95 \% \mathrm{CI}-1.89,0.86)$. There were no significant differences in the changes in $\mathrm{HbA}_{1 \mathrm{c}}$ or fasting glucose between metformin users and non-users in any of the exercise groups compared with control ( $p>0.32$ for all metformin by group by time interactions [Fig. 1]).

Increases in $\dot{V} \mathrm{O}_{2 \text { peak }}$ (expressed in $\mathrm{ml} \mathrm{kg}^{-1} \mathrm{~min}^{-1}$ or $1 / \mathrm{min}$ ) were about twice as large in non-metformin users compared with metformin users following aerobic or combined training (Table 2). However, the metformin by group by time interactions were not significant.

\section{Conclusions}

Contrary to our hypothesis, use of metformin was not associated with smaller improvements in glycaemic control following exercise training. This finding is important because it is contrary to recent studies suggesting that the addition of exercise to metformin treatment increased postprandial glucose [1], increased hepatic glucose output [2] and had no additional effect on insulin sensitivity $[2,3]$ compared with metformin treatment alone. The DARE trial represents the largest supervised exercise study examining this issue and is the only study that includes a group dedicated to performing resistance training alone.
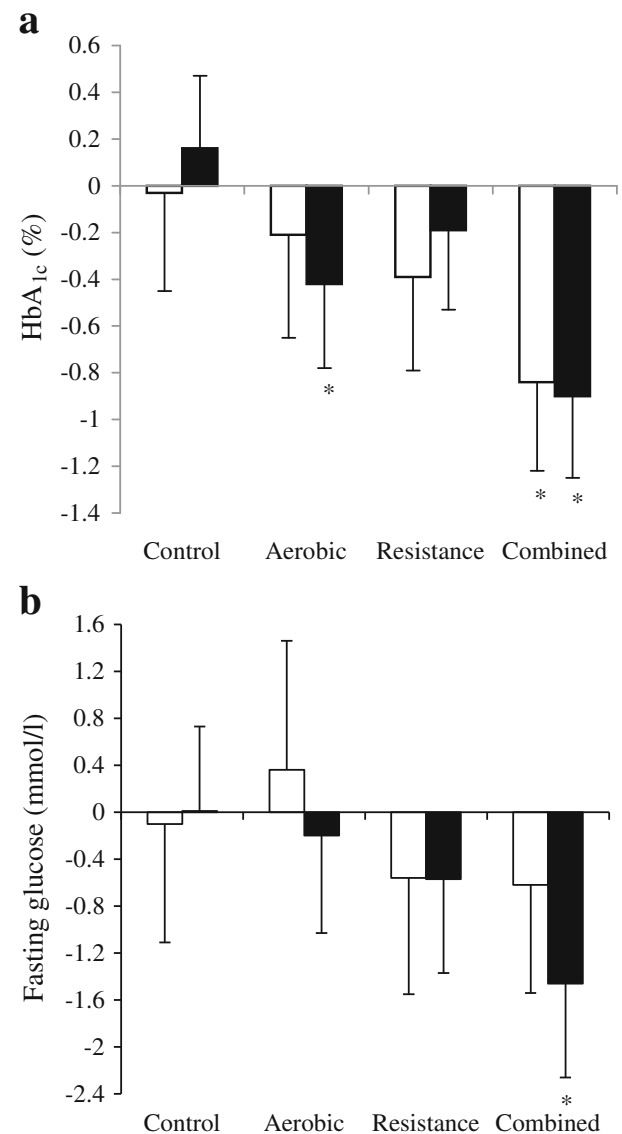

Fig. 1 Effect of exercise, by metformin treatment, on $\mathrm{HbA}_{1 \mathrm{c}}$ (a) and fasting glucose (b). White bars, no metformin; black bars, metformin treatment. The number of participants was 19, 42, 18, 32, 20, 35, 25, 34 in the groups from left to right. Results are shown as mean changes and $95 \%$ CIs adjusted for (age, sex, BMI and site) from mixed models on the data stratified by metformin use. ${ }^{*} p<0.05$ vs corresponding Control group. None of the contrast estimates from the mixed model for the metformin by group by time interaction were statistically significant (all $p>0.32$ )

There are differences between the present study and previous ones [1-3] that may help explain the disparate results. The most important differences may relate to the timing and type of measures of glycaemic control or insulin sensitivity. In previous studies [1-3] meal tolerance tests or hyperinsulinaemiceuglycaemic clamps were performed within $28 \mathrm{~h}$ of an exercise session. In contrast, $\mathrm{HbA}_{1 \mathrm{c}}$ reflects average blood glucose concentration over the previous 2-3 months and fasting glucose measurements in DARE had been taken at least 2 days after the last exercise session.

Alternatively, it could be speculated that differences were due to the fact that previous studies were performed in metformin-naive participants [1-3] whereas participants in the DARE trial had been taking metformin for a longer time before starting exercise. The previous studies involved randomly assigned participants with better glycaemic control (i.e. insulin resistant without diabetes [2], impaired glucose tolerance [3] and diabetes with mean $\mathrm{HbA}_{1 \mathrm{c}}$ of $6.5 \%$ (48 $\left.\mathrm{mmol} / \mathrm{mol}\right)$ [1]). 


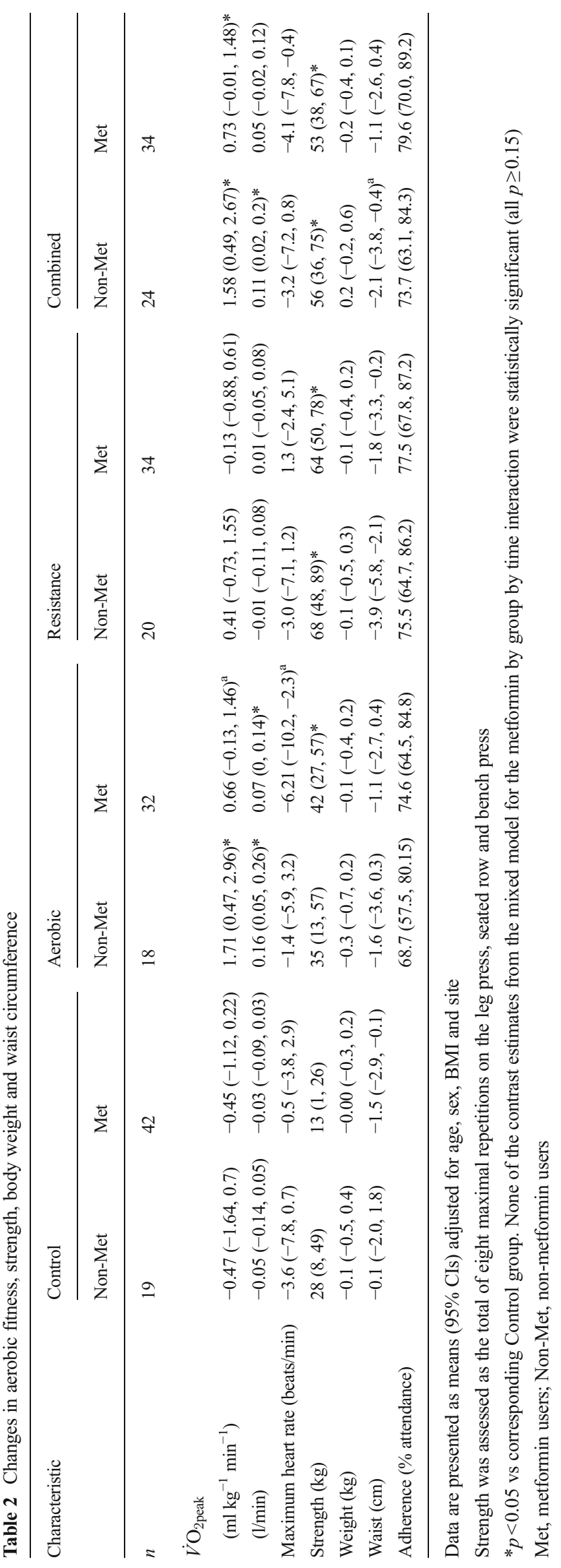


It is possible that not all individuals are affected similarly by interactions between metformin and exercise.

Changes in indicators of fitness were not significantly affected by metformin. However, the difference between metformin users and non-users was in the same direction as in previous studies, suggesting that metformin reduces improvements in aerobic fitness [3, 4].

The primary limitation of the present analyses was the absence of randomisation to metformin or placebo. The impact of confounders was minimised through the inclusion of a control group and by adjusting for differences such as age, sex and BMI. Baseline $\mathrm{HbA}_{1 \mathrm{c}}$ level is known to be directly related to the magnitude of the improvements in $\mathrm{HbA}_{1 \mathrm{c}}$ levels following exercise [8]. Baseline $\mathrm{HbA}_{1 \mathrm{c}}$ was higher and there were greater proportions of men in the subgroup of metformin users. This may in part explain why the patients treated with metformin tended to respond more favourably following aerobic training. The relatively good glycaemic control at baseline in the DARE trial may have also constrained the magnitude of the intervention effects [8].

Other glucose-lowering medications were used by participants in the DARE trial [6] and metformin users were often also treated with them. It would have been interesting to examine the effects of other medications. However, metformin was chosen since it was the most commonly used medication in DARE participants and the study was underpowered to examine interactions among several medications.

In summary, metformin did not significantly affect improvements in $\mathrm{HbA}_{1 \mathrm{c}}$ and fasting glucose, fitness and anthropometrics resulting from 6 months of aerobic, resistance or combined aerobic and resistance training.

Funding The DARE trial was supported by grants from the Canadian Institutes of Health Research (MCT-44155) and the Canadian Diabetes Association (The Lillian Hollefriend Grant). The funding sources had no role in design, conduct or reporting of the study.
Duality of interest The authors declare that there is no duality of interest associated with this manuscript.

Contribution statement NGB, GPK and RJS were responsible for the conception and design of the study. Analysis and interpretation of the data was carried out by NGB, GPK, JL, FK, NK and RJS. NGB drafted the article and NGB, GPK, JL, FK, NK and RJS critically revised the article for important intellectual content. Statistical expertise was provided by FK. GPK and RJS obtained funding. Collection and assembly of data was carried out by NGB, GPK and JL. All authors gave final approval for the article to be published.

\section{References}

1. Boulé NG, Robert C, Bell GJ et al (2011) Metformin and exercise in type 2 diabetes: examining treatment modality interactions. Diabetes Care 34:1469-1474

2. Sharoff CG, Hagobian TA, Malin SK et al (2010) Combining short-term metformin treatment and one bout of exercise does not increase insulin action in insulin-resistant individuals. Am J Physiol Endocrinol Metab 298:E815-E823

3. Malin SK, Gerber R, Chipkin SR, Braun B (2012) Independent and combined effects of exercise training and metformin on insulin sensitivity in individuals with prediabetes. Diabetes Care 35:131-136

4. Braun B, Eze P, Stephens BR et al (2008) Impact of metformin on peak aerobic capacity. Appl Physiol Nutr Metab 33:61-67

5. Johnson ST, Robert C, Bell GJ, Bell RC, Lewanczuk RZ, Boule NG (2008) Acute effect of metformin on exercise capacity in active males. Diabetes Obes Metab 10:747-754

6. Sigal RJ, Kenny GP, Boule NG et al (2007) Effects of aerobic training, resistance training, or both on glycemic control in type 2 diabetes: a randomized trial. Ann Intern Med 147:357369

7. Larose J, Sigal RJ, Boule NG et al (2010) Effect of exercise training on physical fitness in type II diabetes mellitus. Med Sci Sports Exerc 42:1439-1447

8. Umpierre D, Ribeiro PA, Kramer CK et al (2011) Physical activity advice only or structured exercise training and association with $\mathrm{HbA} 1 \mathrm{c}$ levels in type 2 diabetes: a systematic review and meta-analysis. JAMA 305:1790-1799 